Bull. Chem. Soc. Ethiop. 2013, 27(1), 85-94.

Printed in Ethiopia

DOI: http://dx.doi.org/10.4314/bcse.v27i1.9

ISSN 1011-3924

(c) 2013 Chemical Society of Ethiopia

\title{
USE OF TRANSESTERIFIED 1,3-DIKETOESTERS IN THE SYNTHESIS OF TRISUBSTITUTED PYRAZOLES AND THEIR BIOLOGICAL SCREENING
}

\author{
Naqui-Jahan Siddiqui $^{1 *}$, Mohammad Idrees ${ }^{1}$, Niraj T. Khati ${ }^{2}$ and Madhukar G. Dhonde ${ }^{3}$ \\ ${ }^{1}$ Department of Chemistry, Government Science College, Gadchiroli - 442605 (M.S.), India \\ ${ }^{2}$ Department of Applied Chemistry, Priyadarshini Engineering College, Nagpur (M.S.), India \\ ${ }^{3}$ Department of Chemistry, Shri Mathuradas Mohata College of Science, Nagpur (M.S.), India
}

(Received May 14, 2012; revised November 15, 2012)

\begin{abstract}
Starting from 2-acetylbenzofuran derivatives 1a-d, methyl/ethyl 4-substituted/unsubstituted benzofuran-2-yl)-2,4-dioxobutanoate 2a-d and 3a-d have been synthesized by Claisen's condensation reaction with diethyloxalate. The transesterified product, 1,3-diketoester 2a-d on condensation with phenyl hydrazine undergo cyclization to afford the corresponding methyl 5-(substituted/unsubstituted benzofuran-2-yl)-1-phenyl$1 \mathrm{H}$-pyrazole-3-carboxylate 4a-d, which upon further condensation with hydrazine hydrate yielded 5(substituted/unsubstituted benzofuran-2-yl)-1-phenyl-1H-pyrazole-3-carbohydrazide 5a-d. The structures of the newly synthesized compounds 2a-d, 3a-d, 4a-d and 5a-d were characterized by their elemental analysis and spectral studies such as IR, ${ }^{1} \mathrm{H}$ NMR, ${ }^{13} \mathrm{C}$ NMR and MS. All the synthesized compounds were screened for their antimicrobial activity. Most of the synthesized compounds showed high sensitivity against the selected bacteria and fungi at various concentrations.
\end{abstract}

KEY WORDS: 2,4-Dioxobutanoate, Prazole-3-carboxylate, Pyrazole-3-carbohydrazide

\section{INTRODUCTION}

As 1,3-diketoester derivatives have been found very reactive towards organic reagent such as hydrazine hydrate, phenyl hydrazine, semicarbazide hydrochloride, hydroxyl amine hydrochloride and hence utilized for the synthesis of substituted pyrazole derivatives[1-3]. Pyrazoles are important class of nitrogen containing five membered heterocyclic compounds. Compounds with pyrazole ring are of interest due to their broad spectrum of biological activities like antibacterial [4, 5], antiameobic [6], fungicidal [7, 8], antidiuretic [9], anticancer [10], potent antidiabetic agent [11], anti-inflammatory [12], antidepressant [13], and antiviral [14]. Moreover N-phenyl pyrazole derivatives play an important role in antitumor screening [15] as well as potent antimicrobial activity $[16,17]$. Some substituted pyrazoles also exhibits cyclooxygenes-2-(Cox2) selective inhibitors $[18,19]$. Literature survey indicated that the hydrazone group plays an important role for the antimicrobial activity. A number of hydrazide-hydrazone derivatives also have been claimed to possess interesting bioactivity such as antibacterialantifungal [20, 21], anti-inflammatory [22], antimalarial [23], anticonvulsant [24], antituberculosis [25, 26], and anticancer [27] activities. So a few pyrazole carbohydrazide hydrazone derivatives have also been reported, which have been synthesized by many methods [28-30]. Encouraged by the importance of pyrazole rings in various pharmacological lead molecules we thought of incorporating this moiety to our base material for research. As a part of our continuing interest in heterocyclic chemistry we turned our attention with the aim to synthesize and evaluate the antimicrobial activities of different trisubstituted pyrazole derivatives derived from methyl 4-(substituted/unsubstituted benzofuran-2-yl)-2,4dioxobutanoate as a stating material.

*Corresponding author. E-mail: naquiphd.2010@gmail.com 


\section{RESULTS AND DISCUSSION}

The synthesis of the novel compounds 2a-d, 3a-d, 4a-d and 5a-d is described in reaction scheme. At every stage the purity of the compounds were monitored by TLC technique. The identities of the newly synthesized compound have been established on the basis of their elemental analysis and spectral data such as IR, ${ }^{1} \mathrm{H}$ NMR, ${ }^{13} \mathrm{C}$ NMR and mass spectral studies. All the obtained products were screened for their antimicrobial activities.

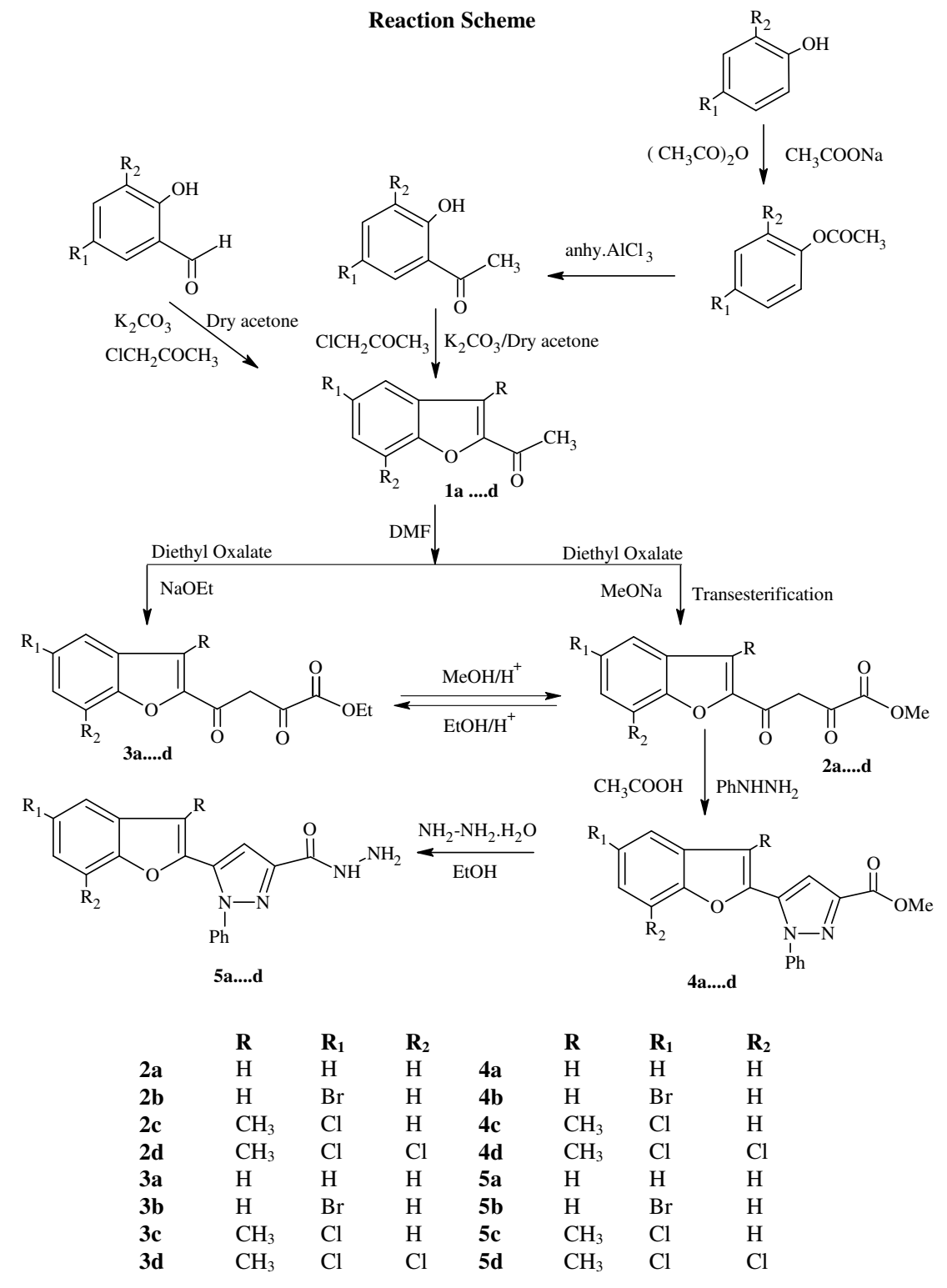

Bull. Chem. Soc. Ethiop. 2013, 27(1) 
The syntheses of the key intermediates 2-acetyl substituted/unsubstituted benzofuran 1a-d were prepared in quantitative yields according to the reference method [31]. The reaction of 1ad with diethyl oxalate in presence of sodium methoxide solution as a base and DMF as solvent afforded 2a-d via transesterification, while the same reaction with sodium ethoxide furnished 3a-d. The ${ }^{1} \mathrm{H}$ NMR and IR spectra of $\mathbf{2 a - d}$ exhibited characteristic band of enolic group due to keto-enol tautomerism of reactive methylene group which was also confirmed by the test with alcoholic $\mathrm{FeCl}_{3}$ which gave wine red colouration. The IR spectrum of methyl 4-(benzofuran-2yl)-2,4-dioxobutanoate 2 a showed the $-\mathrm{OH}$ stretch of enol form at $3475 \mathrm{~cm}^{-1}$ and $\mathrm{C}=\mathrm{O}$ stretching in ester group at $1759 \mathrm{~cm}^{-1}$. The ${ }^{1} \mathrm{H}$ NMR spectrum showed singlet signal at $\delta 14.24$ ppm for one proton of $-\mathrm{OH}$ group, confirms the enolic form, singlet signal at $\delta 3.95 \mathrm{ppm}$ due to $\mathrm{OCH}_{3}$ confirms the methyl ester and multiplet signals at $\delta 7.20-7.71 \mathrm{ppm}$ for five aromatic protons and singlet at $\delta 7.10 \mathrm{ppm}$ confirms one proton due to the vinylic $=\mathrm{CH}$. The chemical shift value of the methoxy carbon in ${ }^{13} \mathrm{C}$ NMR is observed at $\delta 53 \mathrm{ppm}\left(-\mathrm{O} \underline{\mathrm{C}} \mathrm{H}_{3}\right)$, the carbon atoms connected to methoxy group are observed at the $\delta 156-167 \mathrm{ppm}$ range, signal $\delta 167 \mathrm{ppm}$ is due to $\mathrm{C}_{1}$ carbon in $\mathrm{C}=\mathrm{O}$ of the ester group whereas $\mathrm{C}_{4}$ carbon in $\mathrm{C}=\mathrm{O}$ group under the influence of strong electronegative environment appears downfield at $\delta 181 \mathrm{ppm}$, the aromatic carbons were observed in expected region. The mass spectrum [32] of this product reveals a molecular ion at $\mathrm{m} / z 247[\mathrm{M}+\mathrm{H}]^{+}$and $269[\mathrm{M}+\mathrm{Na}]^{+}$is in consistent with the molecular formula $\mathrm{C}_{13} \mathrm{H}_{10} \mathrm{O}_{5}$.

The IR spectra of ethyl 4-[5-chloro-3-methylbenzofuran-2-yl)-2,4-dioxobutanoate 3c showed $-\mathrm{OH}$ stretch at $3632 \mathrm{~cm}^{-1}$ and $\mathrm{C}=\mathrm{O}$ stretching in ester group at $1724 \mathrm{~cm}^{-1}$, respectively. The ${ }^{1} \mathrm{H}$ NMR spectrum showed singlet signal at $\delta 14.79 \mathrm{ppm}$ due to one proton confirms the $\mathrm{OH}$ group in the enolic form of $-\mathrm{OHC}=\mathrm{CH}$ - due to keto-enol tautomerism, triplet signal at $\delta$ 1.41-1.44 ppm for two proton of $-\mathrm{CH}_{2}$ group, quartet signal at $\delta 4.38-4.44 \mathrm{ppm}$ confirms $-\mathrm{OCH}_{2} \mathrm{CH}_{3}$ the ethyl ester and multiplet signals at $\delta$ 7.26-7.52 ppm for three aromatic protons and singlet at $\delta 7.11 \mathrm{ppm}$ confirms one proton due to the vinylic $=\mathrm{CH}$ of $\mathrm{OHC}=\mathrm{CH}-$. The chemical shift values of the ethoxy carbons in ${ }^{13} \mathrm{C}$ NMR spectrum is observed at $\delta 14 \mathrm{ppm}$ $\left(-\mathrm{OCH}_{2} \mathrm{CH}_{3}\right)$ and $62 \mathrm{ppm}\left(-\mathrm{OCH}_{2} \mathrm{CH}_{3}\right)$, the carbon atoms connected to ethoxy group are observed at the $\delta 152-167 \mathrm{ppm}$ range, $\mathrm{C}_{4}$ carbon in $\mathrm{C}=\mathrm{O}$ group appears at $\delta 183 \mathrm{ppm}$ while signal at $\delta 167 \mathrm{ppm}$ is due to $\mathrm{C}_{1}$ of $\mathrm{C}=\mathrm{O}$ carbon of the ester group. The molecular ion peak at $m / z, 309 \mathrm{M}^{+}, 331\left[(\mathrm{M}+\mathrm{Na})^{+},{ }^{35} \mathrm{Cl}\right], 333\left[(\mathrm{M}+\mathrm{Na})^{+},{ }^{37} \mathrm{Cl}\right]$; is in agreement with the molecular formula $\mathrm{C}_{15} \mathrm{H}_{13} \mathrm{O}_{5} \mathrm{Cl}$.

2a-d on reaction with phenyl hydrazine gave corresponding 4a-d. The IR spectra of methyl 5-(benzofuran-2-yl)-1-phenyl-1H-pyrazole-3-carboxylate 4a showed absorption band in the region $1734 \mathrm{~cm}^{-1}$ due to $\mathrm{C}=\mathrm{O}$ stretching in ester group. The characteristic band at $1618 \mathrm{~cm}^{-1}$ shows strong $\mathrm{C}=\mathrm{N}$ stretching bands. This is in evidence with the ring closure of pyrazole ring. The ${ }^{1} \mathrm{H}$ NMR spectrum showed singlet signal at $\delta 3.97 \mathrm{ppm}$ due to three proton confirms the $-\mathrm{COOCH}_{3}$ group in the ester, multiplet at $\delta 7.17-7.54 \mathrm{ppm}$ for ten aromatic proton and one singlet signals at $\delta 6.22 \mathrm{ppm}$ for one proton of pyrazole ring. ${ }^{13} \mathrm{C}$ NMR spectrum shows that the five membered heterocycle pyrazole is formed via cyclization and its signal is characteristically influenced by the phenyl substituent. The $\mathrm{C}_{3}$ atom of pyrazole is found to resonate at about $\delta$ $145 \mathrm{ppm}$ while $\mathrm{C}_{5}$ atom at $\delta 154 \mathrm{ppm}$, signal at $\delta 162 \mathrm{ppm}$ is due to the $\mathrm{C}=\mathrm{O}$ carbon atom of ester and methoxy carbon $\left(-\mathrm{OCH}_{3}\right)$ gives signal at $\delta 53 \mathrm{ppm}$. Similarly the mass spectra reveals a molecular ion peak at $\mathrm{m} / \mathrm{z} 319[\mathrm{M}+\mathrm{H}]^{+}$and $341[\mathrm{M}+\mathrm{Na}]^{+}$is same as the molecular formula $\mathrm{C}_{19} \mathrm{H}_{14} \mathrm{O}_{3} \mathrm{~N}_{2}$.

Formulation of the reaction product designed as 4a-d, was based upon the comparative reactivity of two carbonyl groups in 2a-d. The $\mathrm{C}_{2}$ carbonyl group being more reactive than $\mathrm{C}_{4}$ carbonyl group [1], it gets preferably attacked by the nucleophilic reagent such as phenyl hydrazine to give corresponding hydrazone intermediate which simultaneously undergo ring 
closure with elimination of water molecule from imino proton of hydrazone residue and the $-\mathrm{OH}$ group of enolized $\mathrm{C}_{4}$ carbonyl group.

The reaction of 4a-d, with hydrazine hydrate in ethanol gave 5a-d. Its structure was supported by IR revealing the presence of $\mathrm{C}=\mathrm{O}$ group and $-\mathrm{NH}$ stretch. The IR spectra of 5(benzofuran-2-yl)-1-phenyl-1H-pyrazole-3-carbohydrazide 5a showed characteristic absorption band at $3429 \mathrm{~cm}^{-1}$ indicates the stretch due to $-\mathrm{NHNH}_{2}$ and $\mathrm{C}=\mathrm{O}$ stretch in $-\mathrm{CONHNH}_{2}$ at $1683 \mathrm{~cm}^{-1}$ and $\mathrm{C}=\mathrm{N}$ stretch in pyrazole ring at $1649 \mathrm{~cm}^{-1}$, respectively. The ${ }^{1} \mathrm{H}$ NMR spectrum showed singlet signal at $\delta 3.69-3.71 \mathrm{ppm}$ due to two proton of $-\mathrm{NH}_{2}$ in $-\mathrm{CONHN \underline {H } _ { 2 }}$ and a broad band at $\delta 8.49 \mathrm{ppm}$ for one proton of $-\mathrm{NH}$ in $\mathrm{CON}^{\mathrm{HNH}}{ }_{2}$ and multiplet signals at $\delta$ 7.17-7.51 ppm for ten aromatic protons and singlet at $\delta 6.22 \mathrm{ppm}$ is due to one proton of pyrazole. $\mathrm{In}{ }^{13} \mathrm{C}$ NMR spectrum, the absence of signal corresponding to methoxy carbon as in $4 \mathbf{a}-\mathbf{d}$ at $\delta 53 \mathrm{ppm}$ clearly indicates that ester group has been converted to carbohydrazide $-\mathrm{CONHNH}_{2}, \mathrm{C}=\mathrm{O}$ carbon of carbohydrazide gives signal at $\delta 162 \mathrm{ppm}$ while the aromatic carbons, $\mathrm{C}_{3}$ and $\mathrm{C}_{5}$ in the pyrazole ring are at their expected regions. The elemental analysis of this product gave $C, 67.50$; $\mathrm{H}, 4.35$; N, 16.88; and mass spectrum reveals a molecular ion at $\mathrm{m} / \mathrm{z} 319[\mathrm{M}+\mathrm{H}]^{+}$and 341 $[\mathrm{M}+\mathrm{Na}]^{+}$is in consistent with the molecular formula $\mathrm{C}_{18} \mathrm{H}_{14} \mathrm{O}_{2} \mathrm{~N}_{4}$.

The structures of the other novel synthesized compounds, 2b-d, 3b-d, $4 \mathbf{b}-\mathbf{d}$ and $\mathbf{5 b}$-d were also confirmed by $\mathrm{CHN}$ and spectral investigation such as IR, ${ }^{1} \mathrm{H}$ NMR, ${ }^{13} \mathrm{C}$ NMR, and mass spectra. Simultaneously, the physical constant, yield, spectroscopic and analytical data are also mentioned.

\section{Antimicrobial activity}

The investigation of the microbial screening data revealed that all the tested compounds showed variable activities towards the fungus and bacteria used, which showed that these compounds are biologically active due to the presence of different heterocycles and functional groups. The test compound $\mathbf{2 a}, \mathbf{2 b}, \mathbf{2 d}, \mathbf{3 a}, \mathbf{3 b}, \mathbf{3 c}, \mathbf{3 d}, \mathbf{4 a}, \mathbf{4 c}, \mathbf{4 d}, \mathbf{5 b}, \mathbf{5 c}$ were found to possess moderate to high activity, whereas $\mathbf{2 c}, \mathbf{4 b}, \mathbf{4 c}, \mathbf{5 a}, \mathbf{5 d}$ were found to be poorly active at $300-500 \mu \mathrm{g} / \mathrm{mL}$, but inactive at 100 or $200 \mu \mathrm{g} / \mathrm{mL}$ concentration against the fungus Aspergillus niger as given in the Table 1. Similarly the result of antibacterial activity are also tabulated which clearly indicates that the synthesized compounds $\mathbf{2 a}, \mathbf{3 a}, \mathbf{3 b}, \mathbf{4 a}, \mathbf{4 b}, \mathbf{4 d}, \mathbf{5 a}$, are highly active, compounds $\mathbf{2 b}, \mathbf{3 c}$, $4 \mathbf{c}, 5 \mathbf{b}, \mathbf{5 c}$ are moderately active while $\mathbf{2 c}, \mathbf{2 d}, \mathbf{3 d}, \mathbf{5 d}$ are poorly active against $S$. aureus. Compounds $\mathbf{3 b}, \mathbf{3 c}, \mathbf{4 a}, \mathbf{5 b}, \mathbf{5 c}$ are highly active, $\mathbf{2 b}, \mathbf{2 c}, \mathbf{2 d}, \mathbf{3 a}, \mathbf{3 d}, \mathbf{4 c}, \mathbf{5 a}$ are moderately active and $\mathbf{2 a}, \mathbf{4 b}, \mathbf{4 d}$ and $\mathbf{5 d}$ are poorly active against $E$. coli.

\section{EXPERIMENTAL}

The melting points were recorded in open capillary in paraffin bath and are uncorrected. IR spectra were recorded on a Shimadzu IR Spectrophotometer $\left(\mathrm{KBr}, v\right.$ max in $\left.\mathrm{cm}^{-1}\right) .{ }^{1} \mathrm{H}$ NMR and ${ }^{13} \mathrm{C}$ NMR spectra are recorded on a Bruker AM 400 instrument (400 MHz) using tetramethylsilane (TMS) as an internal reference and DMSO- $\mathrm{d}_{6}$ and $\mathrm{CDCl}_{3}$ as solvent. Chemical shifts are given in parts per million ( $\mathrm{ppm}$ ). Positive-ion electrospray ionisation (ESI) mass spectra were obtained with a Waters Micromass Q-TOF Micro, Mass Spectrophotometer. Elemental analysis (CHN) was done using Elemental analyzer, Vario EL III. All the chemicals used for the synthesis were of AR grade of Merck, S.D. Fine and Aldrich. The reactions were monitored by $\mathrm{E}$. Merck TLC aluminum sheet silica gel $_{60} \mathrm{~F}_{254}$ and visualizing the spot in UV cabinet and iodine chamber. The antimicrobial screening of the synthesized compounds were carried out at microbiology laboratory. 
Table 1. Antimicrobial activity of the synthesized compounds $\mathbf{2 a - d , ~ 3 a - d , ~ 4 a - d ~ a n d ~ 5 a - d . ~}$

\begin{tabular}{|c|c|c|c|c|c|c|c|c|}
\hline \multirow{3}{*}{ No. } & \multirow{3}{*}{ Compound } & \multicolumn{5}{|c|}{ Antifungal activity } & \multicolumn{2}{|c|}{ *Antibacterial activity } \\
\hline & & \multicolumn{7}{|c|}{ Zone of inhibition in $\mathrm{mm}$} \\
\hline & & \multicolumn{5}{|c|}{ A. niger } & S. aureus & E. coli \\
\hline \multirow{2}{*}{\multicolumn{2}{|c|}{ Concentration }} & \multicolumn{5}{|c|}{$\mu \mathrm{g} / \mathrm{mL}$} & \multirow{2}{*}{\multicolumn{2}{|c|}{$1 \mathrm{mg} / \mathrm{mL}$}} \\
\hline & & 100 & 200 & 300 & 400 & 500 & & \\
\hline 1 & $2 \mathbf{a}$ & 7 & 9 & 9 & 11 & 12 & 26 & 11 \\
\hline 2 & $2 \mathbf{b}$ & 8 & 9 & 11 & 11 & 13 & 21 & 14 \\
\hline 3 & $2 c$ & - & - & 7 & 9 & 11 & 15 & 12 \\
\hline 4 & $2 d$ & 7 & 7 & 8 & 9 & 11 & 16 & 12 \\
\hline 5 & $3 \mathbf{a}$ & 9 & 11 & 15 & 16 & 16 & 25 & 14 \\
\hline 6 & $3 \mathbf{b}$ & 9 & 11 & 13 & 15 & 17 & 28 & 16 \\
\hline 7 & $3 c$ & 7 & 9 & 10 & 13 & 16 & 21 & 15 \\
\hline 8 & $3 d$ & 8 & 8 & 10 & 12 & 14 & 17 & 13 \\
\hline 9 & $4 \mathbf{a}$ & 7 & 9 & 9 & 10 & 11 & 28 & 17 \\
\hline 10 & $4 b$ & - & - & 7 & 7 & 9 & 24 & 10 \\
\hline 11 & $4 c$ & - & 7 & 9 & 10 & 14 & 20 & 13 \\
\hline 12 & 4d & 7 & 7 & 8 & 8 & 9 & 25 & 11 \\
\hline 13 & $5 \mathbf{a}$ & - & 6 & 7 & 8 & 9 & 26 & 12 \\
\hline 14 & $5 \mathbf{b}$ & 8 & 9 & 10 & 12 & 14 & 20 & 17 \\
\hline 15 & $5 c$ & - & 9 & 10 & 12 & 15 & 21 & 18 \\
\hline 16 & $5 d$ & - & - & - & 8 & 10 & 13 & 10 \\
\hline \multicolumn{2}{|c|}{ Kanamycin } & 8 & 9 & 14 & 18 & 23 & - & - \\
\hline \multicolumn{2}{|c|}{ Chloramphenicol } & - & - & - & - & - & 32 & 21 \\
\hline \multicolumn{2}{|c|}{ DMSO } & - & - & - & - & - & - & - \\
\hline
\end{tabular}

General procedure for the synthesis of (1a-d)

2-Hydroxy acetophenone/2-hydroxy benzaldehyde derivatives $(10 \mathrm{mmol})$ were taken in dry acetone $(40 \mathrm{~mL})$ and chloroacetone $(10 \mathrm{mmol})$ was added dropwise at room temperature for $1 \mathrm{~h}$. Then freshly ignited $\mathrm{K}_{2} \mathrm{CO}_{3}(15 \mathrm{mmol})$ was added, the reaction mixture was refluxed on steam bath for $8 \mathrm{~h} . \mathrm{K}_{2} \mathrm{CO}_{3}$ was removed by washing with acetone. This combined acetone extract was distilled on reduced pressure then cooled and kept overnight, product obtained was filtered, washed with water, dried and recrystallized from ethanol [31].

\section{General procedure for the synthesis of $(2 a-d)$}

To a solution of 1a-d $(10 \mathrm{mmol})$ and sodium methoxide $(10 \mathrm{mmol})$ in DMF $(100 \mathrm{~mL})$, diethyloxalate $(10 \mathrm{mmol})$ was gradually added with shaking. The reaction mixture was then stirred for $12 \mathrm{~h}$ at room temperature; the product so obtained was acidified by 1:1 ice-cold $\mathrm{HCl}$, filtered, washed with water and recrystallized from suitable solvent.

Methyl 4-(benzofuran-2-yl)-2,4-dioxobutanoate (2a) [33]. Yellow crystals, yield: (85\%); m.p.:131-133 ${ }^{0} \mathrm{C}$ (from DMF or acetone); IR (KBr, $v$ in $\left.\mathrm{cm}^{-1}\right) 3475(-\mathrm{OH}), 3059,3020$ (ArH), 2968, $2879\left(\mathrm{CH}_{3}\right)$ 1805, $1759(\mathrm{C}=\mathrm{O}$, ester $), 1624,1573,1521(\mathrm{C}=\mathrm{C}) ;{ }^{1} \mathrm{H}$ NMR $\left(\mathrm{CDCl}_{3}\right) \delta$ (ppm): 3.95 (s, 3H, $\left.\mathrm{CH}_{3}\right), 7.20-7.71(\mathrm{~m}, 5 \mathrm{H}, \mathrm{ArH}), 7.19(\mathrm{~s}, 1 \mathrm{H},=\mathrm{CH}), 14.24(\mathrm{~s}, 1 \mathrm{H},-\mathrm{OH}) ;{ }^{13} \mathrm{C}$ NMR $\delta$ (ppm): 53, 99, 112, 114, 123, 124, 127, 128, 150, 156, 162, 167, 181; ESI(+)-MS: $\mathrm{m} / \mathrm{z}$ $247(\mathrm{M}+\mathrm{H})^{+}, 269(\mathrm{M}+\mathrm{Na})^{+}$; anal. calcd. for $\mathrm{C}_{13} \mathrm{H}_{10} \mathrm{O}_{5}: \mathrm{C}, 63.41 ; \mathrm{H}, 4.06$; found: $\mathrm{C}, 62.52 ; \mathrm{H}$, 4.13 . 
Methyl 4-(5-bromobenzofuran-2-yl)-2,4-dioxobutanoate (2b). Yellow crystals, yield: (79\%); m.p.: $170-171{ }^{0} \mathrm{C}$ (from DMF or acetone); IR (KBr, $v$ in $\left.\mathrm{cm}^{-1}\right), 3450,3119(-\mathrm{OH}), 3086,3022$ $(\mathrm{ArH}), 2962,2879\left(\mathrm{CH}_{3}\right), 1898,1732(\mathrm{C}=\mathrm{O}$ ester $), 1620,1570(\mathrm{C}=\mathrm{C}) ;{ }^{1} \mathrm{H}$ NMR $\left(\mathrm{CDCl}_{3}\right) \delta$ (ppm): $3.96(\mathrm{~s}, 3 \mathrm{H}, \mathrm{CH} 3), 7.20-7.85(\mathrm{~m}, 4 \mathrm{H}, \mathrm{ArH}), 7.10(\mathrm{~s}, 1 \mathrm{H},=\mathrm{CH}), 14.28(\mathrm{~s}, 1 \mathrm{H},-\mathrm{OH})$, ${ }^{13} \mathrm{C}$ NMR $\delta$ (ppm): 53, 99, 112, 113, 117, 125, 129, 131, 151, 154, 162,168, 180; ESI(+)-MS: $m / z 326(\mathrm{M}+\mathrm{H})^{+}, 347\left[(\mathrm{M}+\mathrm{Na})^{+},{ }^{79} \mathrm{Br}\right], 349\left[(\mathrm{M}+\mathrm{Na})^{+},{ }^{81} \mathrm{Br}\right]$; anal. calcd. for $\mathrm{C}_{13} \mathrm{H}_{9} \mathrm{O}_{5} \mathrm{Br}: \mathrm{C}$, 48.00; H, 2.77; found: C, 47.89; H, 2.59 .

Methyl 4-[5-chloro-3-methylbenzofuran-2-yl)-2,4-dioxobutanoate (2c). Yellow crystals, yield: (89 \%) m.p.: $178-180{ }^{0} \mathrm{C}$ (from DMF or acetone); IR (KBr, $v$ in $\left.\mathrm{cm}^{-1}\right) 3435,3132(-\mathrm{OH}), 3016$, 3086 (ArH), 2956, $2883\left(\mathrm{CH}_{3}\right), 1898,1770,1728$ (C=O, ester), 1635, $1589(\mathrm{C}=\mathrm{C})$; ${ }^{1} \mathrm{H}$ NMR (DMSO) $\delta(\mathrm{ppm}): 2.58-2.65\left(\mathrm{~s}, 3 \mathrm{H}, \mathrm{CH}_{3}\right), 3.76-3.94\left(\mathrm{~b}, 3 \mathrm{H},-\mathrm{OCH}_{3}\right), 7.46-7.73(\mathrm{~m}, 4 \mathrm{H}, \mathrm{ArH}$, $-\mathrm{OH}), 7.12(\mathrm{~s}, 1 \mathrm{H},=\mathrm{CH}) .{ }^{13} \mathrm{C}$ NMR $\delta(\mathrm{ppm}): 9,53,99,113,120,125,128,130,146,152,162$, 168, 180; $\mathrm{ESI}(+)-\mathrm{MS}: \mathrm{m} / z 296(\mathrm{M}+\mathrm{H})^{+}, 317\left[(\mathrm{M}+\mathrm{Na})^{+},{ }^{35} \mathrm{Cl}\right], 319\left[(\mathrm{M}+\mathrm{Na})^{+},{ }^{37} \mathrm{Cl}\right]$; anal. calcd. for $\mathrm{C}_{14} \mathrm{H}_{11} \mathrm{O}_{5} \mathrm{Cl}$ : C, 56.95; H, 3.73; found: C, 56.84; H, 3.82 .

Methyl 4-[5,7-dichloro-3-methylbenzofuran-2-yl)-2,4-dioxobutanoate (2d). Yellow crystals, yield: (65\%); m.p.: 200-202 ${ }^{0} \mathrm{C}$ (from DMF or acetone); IR (KBr, v, in $\left.\mathrm{cm}^{-1}\right), 3446(-\mathrm{OH})$, 3122, 3078, $3018(\mathrm{ArH}), 2956\left(\mathrm{CH}_{3}\right), 1763,1734(\mathrm{C}=\mathrm{O}$, ester $), 1635,1579,1597(\mathrm{C}=\mathrm{C}) ;{ }^{1} \mathrm{H}$ NMR $\left(\mathrm{CDCl}_{3}\right) \delta(\mathrm{ppm}): 2.6\left(\mathrm{~s}, 3 \mathrm{H}, \mathrm{CH}_{3}\right), 3.9\left(\mathrm{~s}, 3 \mathrm{H},-\mathrm{OCH}_{3}\right), 7.2-7.5(\mathrm{~m}, 3 \mathrm{H}, \mathrm{ArH}), 14.68(\mathrm{~b}$, $1 \mathrm{H},-\mathrm{OH})$; ESI(+)-MS: $m / z 331(\mathrm{M}+2)+351\left[(\mathrm{M}+\mathrm{Na})^{+},{ }^{35} \mathrm{Cl}\right], 353\left[(\mathrm{M}+\mathrm{Na})^{+},{ }^{37} \mathrm{Cl}\right]$; anal. calcd. for $\mathrm{C}_{14} \mathrm{H}_{10} \mathrm{O}_{5} \mathrm{Cl}_{2}$ : C, 51.06; $\mathrm{H}, 3.03$; found: $\mathrm{C}, 50.73 ; \mathrm{H}, 3.31$.

General procedure for the synthesis of $(3 a-d)$

To a solution of 1a-d $(10 \mathrm{mmol})$ and sodium ethoxide $(10 \mathrm{mmol})$ in DMF $(100 \mathrm{~mL})$, diethyloxalate $(10 \mathrm{mmol})$ was gradually added with shaking. The reaction mixture was then stirred for $12 \mathrm{~h}$ at room temperature; the product so obtained was acidified by $1: 1$ ice-cold $\mathrm{HCl}$, filtered, washed with water and recrystallized from suitable solvent.

Ethyl 4-(benzofuran-2-yl)-2,4-dioxobutanoate (3a) [34]. Yellow Crystalline, Yield: (55\%); m.p.: 63-65 ${ }^{0} \mathrm{C}$ (from ethanol); IR (KBr, $v$ in $\mathrm{cm}^{-1}$ ) 3653, $3455(\mathrm{OH}), 3107,3080(\mathrm{ArH}), 2980$, 2937, 2904, $2874\left(\mathrm{CH}_{3}\right), 1786,1724\left(\mathrm{C}=\mathrm{O}\right.$, ester), 1633, $1548(\mathrm{C}=\mathrm{C})$; anal. calcd. for $\mathrm{C}_{14} \mathrm{H}_{12} \mathrm{O}_{5}$ : C, 64.61; H, 4.61; found: C, 64.59; H, 4.60.

Ethyl 4-(5-bromobenzofuran-2-yl)-2,4-dioxobutanoate (3b). Yellow crystals, yield: (60\%); m.p.: $130-131{ }^{0} \mathrm{C}$ (from ethanol); IR (KBr, $v$ in $\mathrm{cm}^{-1}$ ) 3423, $3126(-\mathrm{OH}), 3072$ (ArH), 2987, 2899 $\left(\mathrm{CH}_{3}\right), 1888,1724(\mathrm{C}=\mathrm{O}$, ester $), 1627,1562(\mathrm{C}=\mathrm{C}) ;{ }^{1} \mathrm{H}$ NMR $\left(\mathrm{CDCl}_{3}\right) \delta(\mathrm{ppm}): 1.41-1.44(\mathrm{t}, J=$ $\left.7.12 \mathrm{~Hz}, 3 \mathrm{H},-\mathrm{OCH}_{2} \mathrm{CH}_{3}\right), 4.39-4.44\left(\mathrm{q}, J=7.16 \mathrm{~Hz}, 2 \mathrm{H},-\mathrm{OCH}_{2} \mathrm{CH}_{3}\right), 7.26-7.85(\mathrm{~m}, 4 \mathrm{H}, \mathrm{ArH})$, $7.09(\mathrm{~s}, 1 \mathrm{H},=\mathrm{CH})$; $\mathrm{ESI}(+)-\mathrm{MS}: \mathrm{m} / z 339 \mathrm{M}^{+}, 361\left[(\mathrm{M}+\mathrm{Na})^{+},{ }^{79} \mathrm{Br}\right], 363\left[(\mathrm{M}+\mathrm{Na})^{+},{ }^{81} \mathrm{Br}\right]$; anal. calcd. for $\mathrm{C}_{14} \mathrm{H}_{11} \mathrm{O}_{5} \mathrm{Br}$ : C, 49.55; H, 3.24; found: C, 49.18; H, 3.31 .

Ethyl 4-[5-chloro-3-methylbenzofuran-2-yl)-2,4-dioxobutanoate (3c). Yellow crystals, yield: (60\%); m.p.: $145-147{ }^{0} \mathrm{C}$ (from acetone); IR (KBr, $v$ in $\left.\mathrm{cm}^{-1}\right), 3632,3132$ (-OH), 3095 (ArH), $2999\left(\mathrm{CH}_{3}\right), 1871,1724(\mathrm{C}=\mathrm{O}$, ester $), 1678,1639,1599,1575(\mathrm{C}=\mathrm{C}) ;{ }^{1} \mathrm{H} \mathrm{NMR}\left(\mathrm{CDCl}_{3}\right) \delta$ (ppm): 1.41-1.44 (t, $\left.J=7.12 \mathrm{~Hz}, 3 \mathrm{H},-\mathrm{OCH}_{2} \mathrm{CH}_{3}\right), 4.38-4.44\left(\mathrm{q}, J=7.16 \mathrm{~Hz}, 2 \mathrm{H},-\mathrm{OCH}_{2} \mathrm{CH}_{3}\right)$, $2.59\left(\mathrm{~s}, 3 \mathrm{H}, \mathrm{CH}_{3}\right), 7.11(\mathrm{~s}, 1 \mathrm{H},=\mathrm{CH}), 7.26-7.52(\mathrm{~m}, 3 \mathrm{H}, \mathrm{ArH}), 14.79(\mathrm{~b}, 1 \mathrm{H},-\mathrm{OH}) ;{ }^{13} \mathrm{C} \mathrm{NMR} \delta$ (ppm): 9, 14, 62, 99, 113, 120, 126, 128, 129, 130, 146, 152, 161,167,183; ESI(+)-MS: $m / z, 309$ $\mathrm{M}^{+}, 331\left[(\mathrm{M}+\mathrm{Na}),{ }^{+35} \mathrm{Cl}\right], 333\left[(\mathrm{M}+\mathrm{Na})^{+},{ }^{37} \mathrm{Cl}\right]$; anal. calcd. for $\mathrm{C}_{15} \mathrm{H}_{13} \mathrm{O}_{5} \mathrm{Cl}: \mathrm{C}, 58.25 ; \mathrm{H}, 4.21$; found: $\mathrm{C}, 58.00 ; \mathrm{H}, 4.32$. 
Ethyl 4-[5,7-dichloro-3-methylbenzofuran-2-yl)-2,4-dioxobutanoate (3d). Yellow crystals, yield: (45\%); m.p.: $125-127{ }^{\circ} \mathrm{C}$ (from acetone); IR (KBr, $v$ in $\left.\mathrm{cm}^{-1}\right), 3435,3117(-\mathrm{OH}), 3080$, 3007 ( $\mathrm{ArH}), 2987,2945\left(\mathrm{CH}_{3}\right), 1855,1782,1728$ (C=O, ester), 1683, 1639, 1604, $1577(\mathrm{C}=\mathrm{C})$; ${ }^{1} \mathrm{H} \mathrm{NMR}\left(\mathrm{CDCl}_{3}\right) \delta(\mathrm{ppm}): 1.41-1.45\left(\mathrm{t}, J=7.12 \mathrm{~Hz}, 3 \mathrm{H}, \mathrm{OCH}_{2} \mathrm{CH}_{3}\right), 4.40-4.45(\mathrm{q}, J=7.16$ $\left.\mathrm{Hz}, 2 \mathrm{H},-\mathrm{OCH}_{2} \mathrm{CH}_{3}\right), 2.62\left(\mathrm{~s}, 3 \mathrm{H}, \mathrm{CH}_{3}\right), 7.17(\mathrm{~s}, 1 \mathrm{H},=\mathrm{CH}), 7.47(\mathrm{~d}, 2 \mathrm{H}, \mathrm{ArH}), 7.5(\mathrm{~d}, 1 \mathrm{H}, \mathrm{ArH})$, 14.83 (s, $1 \mathrm{H},-\mathrm{OH})$; anal. calcd. for $\mathrm{C}_{15} \mathrm{H}_{12} \mathrm{O}_{5} \mathrm{Cl}_{2}, \mathrm{C}, 54.71 ; \mathrm{H}, 3.65$; found: $\mathrm{C}, 54.68 ; \mathrm{H}, 3.68$.

\section{General procedure for the synthesis of $(4 a-d)$}

To a mixture of $\mathbf{2 a - d}(10 \mathrm{mmol})$ in $\mathrm{CH}_{3} \mathrm{COOH}(10 \mathrm{~mL})$, phenyl hydrazine $(15 \mathrm{mmol})$ was added and the reaction mixture was refluxed for $4 \mathrm{~h}$. After that it was concentrated, cooled and poured in crushed ice, filtered, dried and recrystallized from suitable solvent.

Methyl 5-(benzofuran-2-yl)-1-phenyl-1H-pyrazole-3-carboxylate (4a). White crystals, yield: (85\%); m.p.: $161-163{ }^{0} \mathrm{C}$ (from acetic acid); IR ( $\mathrm{KBr}, v$ in $\left.\mathrm{cm}^{-1}\right), 3061$ (ArH), $2955\left(\mathrm{CH}_{3}\right), 1618$ $(\mathrm{C}=\mathrm{N}), 1734\left(\mathrm{C}=\mathrm{O}\right.$, ester), 1593, 1500, 1436, $1408(\mathrm{C}=\mathrm{C}) ;{ }^{1} \mathrm{H}$ NMR $\left(\mathrm{CDCl}_{3}\right) \delta(\mathrm{ppm}): 3.97$ (s, $\left.3 \mathrm{H},-\mathrm{COOCH}_{3}\right), 7.17-7.54(\mathrm{~m}, 10 \mathrm{H}, \mathrm{ArH}), 6.22(\mathrm{~s}, 1 \mathrm{H}$, pyrazole $\mathrm{CH}) ;{ }^{13} \mathrm{C}$ NMR $\delta(\mathrm{ppm}): 52$, $105,109,111,121,123,125,126,127,129,136,139,144,145,154,162$; ESI(+)-MS: $m / z, 319$ $(\mathrm{M}+\mathrm{H})^{+}, 341(\mathrm{M}+\mathrm{Na})^{+}$; anal. calcd. for $\mathrm{C}_{19} \mathrm{H}_{14} \mathrm{O}_{3} \mathrm{~N}_{2}: \mathrm{C}, 71.69 ; \mathrm{H}, 4.40 ; \mathrm{N}, 8.81$; found: $\mathrm{C}$, $71.05 ; \mathrm{H}, 4.42 ; \mathrm{N}, 8.42$.

Methyl 5-(5-bromobenzofuran-2-yl)-1-phenyl-1H-pyrazole-3-carboxylate (4b). White crystals, yield: (90\%); m.p.: $188-190{ }^{0} \mathrm{C}$ (from acetic acid); IR (KBr, $v$ in $\left.\mathrm{cm}^{-1}\right), 3146,3001(\mathrm{ArH}), 2953$ $\left(\mathrm{CH}_{3}\right), 1734\left(\mathrm{C}=\mathrm{O}\right.$, ester), $1589(\mathrm{C}=\mathrm{N}) ;{ }^{1} \mathrm{H} \mathrm{NMR}\left(\mathrm{CDCl}_{3}\right) \delta(\mathrm{ppm}): 3.97\left(\mathrm{~s}, 3 \mathrm{H},-\mathrm{COOCH}_{3}\right)$, 7.27-7.54 (m, 9H, ArH), 6.12 (s, 1H, pyrazole $\mathrm{CH}) ;{ }^{13} \mathrm{C}$ NMR $\delta(\mathrm{ppm}): 52,104,109,112,116$, 124, 126, 128, 129, 135, 139, $144,146,153,162$; ESI(+)-MS: $m / z 397 \mathrm{M}^{+}, 419\left[(\mathrm{M}+\mathrm{Na})^{+}\right.$, $\left.{ }^{79} \mathrm{Br}\right], 421\left[(\mathrm{M}+\mathrm{Na}){ }^{+},{ }^{81} \mathrm{Br}\right]$; anal. calcd. for $\mathrm{C}_{19} \mathrm{H}_{13} \mathrm{O}_{3} \mathrm{~N}_{2} \mathrm{Br}$ : C, 57.43; H, 3.27; N, 7.05; found: C, $56.98 ; \mathrm{H}, 3.22 ; \mathrm{N}, 6.62$.

Methyl 5-(5-chloro-3-methylbenzofuran-2-yl)-1-phenyl-1H-pyrazole-3-carboxylate (4c). White crystals, yield: (90\%); m.p.: $148-150{ }^{0} \mathrm{C}$ (from acetic acid); IR $\left(\mathrm{KBr}, v\right.$ in $\left.\mathrm{cm}^{-1}\right) 3151,3072$ $(\mathrm{ArH}), 2995,2951\left(\mathrm{CH}_{3}\right), 1720(\mathrm{C}=\mathrm{O}$, ester $), 1689(\mathrm{C}=\mathrm{N}), 1595,1527(\mathrm{C}=\mathrm{C}) ;{ }^{1} \mathrm{H}$ NMR $\left(\mathrm{CDCl}_{3}\right) \delta(\mathrm{ppm}): 2.08\left(\mathrm{~s}, 3 \mathrm{H}, \mathrm{CH}_{3}\right), 3.99\left(\mathrm{~s}, 3 \mathrm{H},-\mathrm{COOCH}_{3}\right), 7.22-7.4(\mathrm{~m}, 8 \mathrm{H}, \mathrm{ArH}), 6.22(\mathrm{~s}$, $1 \mathrm{H}$, pyrazole $\mathrm{CH}) ;{ }^{13} \mathrm{C}$ NMR $\delta(\mathrm{ppm}): 8,52,112,116,119,124,125,126,128,129,130,133$, 139, 141, 144, 152, 162; ESI(+)-MS: $m / z 389\left[(\mathrm{M}+\mathrm{Na})^{+},{ }^{35} \mathrm{Cl}\right], 391\left[(\mathrm{M}+\mathrm{Na})^{+},{ }^{37} \mathrm{Cl}\right]$; anal. calcd. for $\mathrm{C}_{20} \mathrm{H}_{15} \mathrm{O}_{3} \mathrm{~N}_{2} \mathrm{Cl}$ : C, 65.39; H, 4.08; N, 7.63; found: C, 65.00; H, 4.13; N, 7.34.

Methyl 5-(5,7-dichloro-3-methylbenzofuran-2-yl)-1-phenyl-1H-pyrazole-3-carboxylate (4d). White crystals, yield: $(80 \%)$; m.p.: $149-151{ }^{0} \mathrm{C}$ (from acetic acid); IR $\left(\mathrm{KBr}, \mathrm{v}\right.$ in $\left.\mathrm{cm}^{-1}\right), 3082$ $(\mathrm{ArH}), 2993,2953\left(\mathrm{CH}_{3}\right), 1724,1749(\mathrm{C}=\mathrm{O}$, ester $), 1600(\mathrm{C}=\mathrm{N}), 1579,1552,1523(\mathrm{C}=\mathrm{C}) ;{ }^{1} \mathrm{H}$ NMR $\left(\mathrm{CDCl}_{3}\right) \delta(\mathrm{ppm}): 2.06\left(\mathrm{~s}, 3 \mathrm{H}, \mathrm{CH}_{3}\right), 3.99\left(\mathrm{~s}, 3 \mathrm{H},-\mathrm{COOCH}_{3}\right), 7.26-7.46(\mathrm{~m}, 8 \mathrm{H}, \mathrm{ArH}+$ pyrazole $\mathrm{CH}) ;{ }^{13} \mathrm{C}$ NMR $\delta$ (ppm): 8, 52, 111, 112, 113, 116, 117, 118, 120, 123, 124, 125, 128 , $129,131,133,135,139,142,144,148,162$; ESI(+)-MS: $m / z, 401 \mathrm{M}^{+}, 423\left[(\mathrm{M}+\mathrm{Na})^{+},{ }^{35} \mathrm{Cl}\right], 425$ $\left[(\mathrm{M}+\mathrm{Na})^{+},{ }^{37} \mathrm{Cl}\right]$; anal. calcd. for $\mathrm{C}_{20} \mathrm{H}_{14} \mathrm{O}_{3} \mathrm{~N}_{2} \mathrm{Cl}_{2}$ : C, 59.85; H, 3.49; N, 6.98; found: C, 59.80; H, $3.50 ; \mathrm{N}, 6.45$. 
General procedure for the synthesis of $(5 a-d)$

To a mixture of $\mathbf{4 a - d}(10 \mathrm{mmol})$ in ethanol $(100 \mathrm{~mL})$, hydrazine hydrate $(100 \%, 1.7 \mathrm{~mL})$ was added and refluxed for $8 \mathrm{~h}$. Then it was concentrated, cooled, filtered, washed and recrystallized from suitable solvent.

5-(Benzofuran-2-yl)-1-phenyl-1H-pyrazole-3-carbohydrazide (5a) [35]. White crystals, yield: (88\%); m.p.: $145-146{ }^{0} \mathrm{C}$ (from ethanol). $\mathrm{IR}\left(\mathrm{KBr}, v\right.$ in $\left.\mathrm{cm}^{-1}\right) 3429,3317,3225,3159$ (-NH$\left.\mathrm{NH}_{2}\right), 3066(\mathrm{ArH}), 1683(\mathrm{C}=\mathrm{O}), 1649(\mathrm{C}=\mathrm{N}), 1531,1597(\mathrm{C}=\mathrm{C}) ;{ }^{1} \mathrm{H}$ NMR $\left(\mathrm{CDCl}_{3}\right) \delta(\mathrm{ppm})$ : 3.69-3.71 (s, $\left.2 \mathrm{H},-\mathrm{CONHNH}_{2}\right), 8.49$ (b, $\left.1 \mathrm{H},-\mathrm{CONHNH}_{2}\right), 7.17-7.54(\mathrm{~m}, 10 \mathrm{H}, \mathrm{ArH}), 6.22$ (s, $1 \mathrm{H}$, pyrazole $\mathrm{CH}) ;{ }^{13} \mathrm{C} \mathrm{NMR} \delta(\mathrm{ppm}): 105,107,111,121,123,125,126,127,129,136,139$, 145, 145, 154, 162; ESI(+)-MS: $m / z 319(\mathrm{M}+\mathrm{H})^{+}, 341(\mathrm{M}+\mathrm{Na})^{+}$; anal. calcd. for $\mathrm{C}_{18} \mathrm{H}_{14} \mathrm{O}_{2} \mathrm{~N}_{4}$, C, 67.92; H, 4.40; N, 17.61; found: C, 67.50; H, 4.35; N, 16.88 .

5-(5-Bromobenzofuran-2-yl)-1-phenyl-1H-pyrazole-3-carbohydrazide (5b). White crystals, yield: (90\%); m.p.: 174-176 ${ }^{0} \mathrm{C}$ (from ethanol); IR $\left(\mathrm{KBr}, \mathrm{v}\right.$ in $\left.\mathrm{cm}^{-1}\right), 3323\left(-\mathrm{NHNH}_{2}\right), 3068$ $(\mathrm{ArH}), 1683(\mathrm{C}=\mathrm{O}$, ester $), 1664(\mathrm{C}=\mathrm{N}), 1620,1541,1599(\mathrm{C}=\mathrm{C}) ;{ }^{1} \mathrm{H}$ NMR $\left(\mathrm{CDCl}_{3}\right) \delta(\mathrm{ppm})$ : 3.70-3.72 (b, 2H, -CONHNH $\left.{ }_{2}\right), 8.29$ (b, 1H, $\left.-\mathrm{CONHNH}_{2}\right), 7.27-7.56(\mathrm{~m}, 9 \mathrm{H}, \mathrm{ArH}), 6.16(\mathrm{~s}, 1 \mathrm{H}$, pyrazole $\mathrm{CH}) ;{ }^{13} \mathrm{C}$ NMR $\delta(\mathrm{ppm}): 104,108,112,116,123,128,129,135,139,145,146,153$, 162; ESI(+)-MS: $m / z 397 \mathrm{M}^{+}, 399(\mathrm{M}+2)^{+}, 421\left[(\mathrm{M}+\mathrm{Na})^{+},{ }^{79} \mathrm{Br}\right], 422\left[(\mathrm{M}+\mathrm{Na})^{+},{ }^{81} \mathrm{Br}\right]$; anal. calcd. for $\mathrm{C}_{18} \mathrm{H}_{13} \mathrm{O}_{2} \mathrm{~N}_{4} \mathrm{Br}$ : C, 54.40; H, 3.27; N, 14.11; found: C, 53.51; H, 3.48; N, 13.54 .

5-(5-Chloro-3-methylbenzofuran-2-yl)-1-phenyl-1H-pyrazole-3-carbohydrazide (5c). White crystals, yield: (90\%); m.p.: $159-160{ }^{0} \mathrm{C}$ (from ethanol); IR (KBr, $v$ in $\mathrm{cm}^{-1}$ ) 3313, 3279 $\left(-\mathrm{NHNH}_{2}\right), 3068(\mathrm{ArH}), 1678(\mathrm{C}=\mathrm{O}$, ester $), 1618(\mathrm{C}=\mathrm{N}), 1541,1518(\mathrm{C}=\mathrm{C}) ;{ }^{1} \mathrm{H} \mathrm{NMR}\left(\mathrm{CDCl}_{3}\right)$ $\delta(\mathrm{ppm}): 2.10\left(\mathrm{~s}, 3 \mathrm{H}, \mathrm{CH}_{3}\right), 3.86\left(\mathrm{~b}, 2 \mathrm{H},-\mathrm{CONHNH}_{2}\right), 8.33$ (b, 1H, $\left.-\mathrm{CONHNH}_{2}\right), 7.17-7.46$ (m, $9 \mathrm{H}, \mathrm{ArH}) ;{ }^{13} \mathrm{C}$ NMR $\delta$ (ppm): 8, 110, 112, 116, 119, 124, 125, 128, 129, 130, 133, 139, 141, 145, 152, 162; ESI(+)-MS: $m / z 367 \mathrm{M}^{+}, 389\left[(\mathrm{M}+\mathrm{Na})^{+},{ }^{35} \mathrm{Cl}\right], 391\left[(\mathrm{M}+\mathrm{Na})^{+},{ }^{37} \mathrm{Cl}\right]$; anal. calcd. for $\mathrm{C}_{19} \mathrm{H}_{15} \mathrm{O}_{2} \mathrm{~N}_{4} \mathrm{Cl}: \mathrm{C}, 62.12 ; \mathrm{H}, 4.08 ; \mathrm{N}, 15.25$; found: $\mathrm{C}, 62.28 ; \mathrm{H}, 4.00 ; \mathrm{N}, 14.84$.

5-(5,7-Dichloro-3-methylbenzofuran-2-yl)-1-phenyl-1H-pyrazole-3-carbohydrazide (5d). White crystals, yield: (85\%); m.p.: 199-200 ${ }^{0} \mathrm{C}$ (from acetic acid); $\mathrm{IR}\left(\mathrm{KBr}, \mathrm{v}\right.$ in $\left.\mathrm{cm}^{-1}\right), 3313,3140$ $\left(-\mathrm{NHNH}_{2}\right), 3006(\mathrm{ArH}), 2924\left(\mathrm{CH}_{3}\right), 1714(\mathrm{C}=\mathrm{O}$, ester $), 1660(\mathrm{C}=\mathrm{N}), 1604,1575,1500(\mathrm{C}=\mathrm{C})$; ${ }^{1} \mathrm{H}$ NMR $\left(\mathrm{CDCl}_{3}\right) \delta(\mathrm{ppm}): 2.10\left(\mathrm{~s}, 3 \mathrm{H}, \mathrm{CH}_{3}\right), 3.24\left(\mathrm{~b}, 2 \mathrm{H},-\mathrm{CONHNH}_{2}\right), 8.17(\mathrm{~b}, 1 \mathrm{H}$, $\mathrm{CONHNH}_{2}$ ), 7.2-7.42 (m, 8H, ArH); ${ }^{13} \mathrm{C}$ NMR $\delta(\mathrm{ppm}): 8,109,116,117,124,128,131,132$, 139, 142, 145,148, 159, 162; ESI(+)-MS: $m / z \quad 401 \mathrm{M}^{+}, 403(\mathrm{M}+2)^{+}$; anal. calcd. for $\mathrm{C}_{19} \mathrm{H}_{14} \mathrm{O}_{2} \mathrm{~N}_{4} \mathrm{Cl}_{2}$ : C, 56.85; H, 3.49; N,13.96; found: C, 55.65; H, 3.45; N, 12.74 .

Antifungal activity

Test solution was prepared by dissolving known weight of each compound 2a-d, 3a-d, 4a-d and 5a-d in dimethyl sulphoxide (DMSO) as solvent and diluted suitably to give the resultant concentration of $100,200,300,400,500 \mu \mathrm{g} / \mathrm{mL}$. Whatmann No. 1 sterile paper discs $(6 \mathrm{~mm})$ were impregnated with solution and allowed to dry at room temperature. In vitro antifungal activity was determined by using Sabouraud Dextrose Agar obtained from Himedia Ltd/Mumbai. Twenty four hours old culture of selected fungi, Aspergillus niger was mixed with physiological saline and the turbidity was corrected by adding sterile physiological saline and sub cultured on Sabouraud Dextrose and suspended in sterile distilled water to an absorbance of 0.6 at $450 \mathrm{~nm}$. Petri plates were prepared by pouring $10 \mathrm{~mL}$ Sabouraud Dextrose Agar for fungi containing microbial culture and was allowed to solidify. The discs were then applied and the 
plates were incubated at $28{ }^{\circ} \mathrm{C}$ for $72-96 \mathrm{~h}$ (fungi) and the inhibition zone was measured in four directions and expressed as mean and the results were compared by using Kanamycin as antifungal standard.

Antibacterial study

As the sensitivity was not observed at conc. $<500 \mu \mathrm{g} / \mathrm{mL}$, the antibacterial activity of the test compounds has been screened at concentration of $1 \mathrm{mg} / \mathrm{mL}$ using dimethyl sulphoxide (DMSO) as solvent and chloramphenicol $(100 \mu \mathrm{g} / \mathrm{mL})$ as standard for antibacterial activity against Staphylococcus aureus and Escherichia coli in Mueller Hinton Agar by using cup plate agar diffusion method [36, 37]. $10 \mathrm{~mL}$ of this sterilized agar media were poured into petridishes and allowed to solidify. On the surface of media microbial suspension were spread with the help of sterilized triangular loop. A stainless steel cylinder of $10 \mathrm{~mm}$ diameter (pre-sterilized) was used to bore the cavity. Into these wells were added $0.1 \mathrm{~mL}$ portion of the test compound in the solvent. The drug solution was allowed to diffuse for about an hour into the medium. The plates were incubated at $37{ }^{\circ} \mathrm{C}$ for $24 \mathrm{~h}$. Zone of inhibition observed around the cup after respective incubation was measured with the help of Vernier Calipers. The results of antifungal and antibacterial activity are given in the Table 1 .

\section{CONCLUSION}

In summary, we have demonstrated in the present study that transesterification reaction was succeeded for the conversion of 1a-d to methyl 4-substituted/unsubstituted benzofuran-2-yl)2,4-dioxobutanoate 2a-d; which upon condensation with phenyl hydrazine yielded methyl 5(substituted/unsubstituted benzofuran-2-yl)-1-phenyl-1H-pyrazole-3-carboxylate 4a-d. This method provides an easy and versatile access to cyclize 1,3-diketoester for synthesizing pyrazoles of significant biological interest. 4a-d were easily converted to 5-(substituted/ unsubstituted benzofuran-2-yl)-1-phenyl-1 $H$-pyrazole-3-carbohydrazide 5a-d by reaction with hydrazine hydrate. The structures of synthesized compounds were confirmed by spectroscopic investigation and elemental analysis. The biological screenings of the newly synthesized derivatives were carried out against two bacteria and one fungus and it was concluded that the test compounds revealed the antifungal activity much better than their antibacterial activities at lower concentrations.

\section{ACKNOWLEDGEMENTS}

The authors are thankful to the Principal, Priyadarshini College of Engineering, Nagpur for providing Laboratory facilities and to the Director, SAIF, Punjab University, Chandigarh for providing ${ }^{1} \mathrm{H}$ NMR, ${ }^{13} \mathrm{C}$ NMR and mass spectra, RSIC, CDRI, Lucknow, India for providing $\mathrm{CHN}$ analysis. The authors are also thankful to Dr. Ugale, Head, Department of Pharmacology, College of Pharmacy, Kamptee for antimicrobial screening of the compound.

\section{REFERENCES}

1. Hassan, M.M.; Wojtamis. J. Indian J. Chem. 1985, 24B, 188.

2. Hassan, M.A.; Mohammad, S.I.M.; Hassan, M.F. Indian J. Chem. 1996, 35B, 22.

3. Saleh, R.M. Indian J. Chem. 1991, 30B, 313.

4. Holla, B.S.; Akberali, P.M.; Shivananda, M.K. IL Farmaco 2000, 55, 256.

5. Liu, X.-H.; Zhi, L.-P.; Song, B.-A.; Xu, H.-L. Chem. Res. 2008, 24, 454.

6. Abid, M.; Bhat, A.R.; Athar, F.; Azam, A. Eur. J. Med Chem. 2009, 44, 417. 
7. Mustafa, A.; Hismat, C.A.; Yannis, M.M.J. J. Prakt. Chem. 1970, 312, 1091

8. Elnagdi, M.H.F.; Elmoghayor, M.R.H.; Hatez, E.A.A.; Alnionion, H.H. J. Org. Chem. 1975, 40, 2604.

9. Garg, H.B. J. Med. Chem. 1972, 15, 446.

10. Manna, F.; Chementi, F.; Fioravanti, R.; Bolasco, A. Bioorg. Med. Chem. Lett. 2005, 15, 4632.

11. Ahn, J.H.; Kim, H.M.; Jung, S.H.; Kang, S.K.; Kim, K.R.; Rhea, S.D.; Yong, S.D.; Cheon, H.G.; Kim, S.S. Bioorg. Med. Chem. Lett. 2004, 14, 4461.

12. Ragavan, R.V.; Vijayakumar, V.; Kumari, N.S. Eur. J. Med. Chem. 2010, 45, 1173.

13. Jung, J.C.; Watkins, E.B.; Avery, M.A. Heterocycles 2005, 65, 71.

14. Rashad, A.E.; Hegab, M.I.; Abdel-Megeid, R.E.; Micky, J.A.; Abdel- Megeid, F.M.E. Bioorg. Med. Chem. 2008, 16, 7102.

15. Farag, A.M.; Mayhoub, A.S.; Barakat, S.E.; Bayomi, A.H. Bioorg. Med. Chem. 2008, 16, 881.

16. Farag, A.M.; Mayhoub, A.S.; Barakat, S.E.; Bayomi, A.H. Bioorg. Med. Chem. 2008, 16, 4569.

17. Hui, Y.; Ptak, R.; Paulman, R.; Pallansch, M.; TomChanger, C.W. Tetrahedron Lett. 2002, 43, 9255.

18. Ezawa, M.; Garvey D.S.; Janero D.R.; Khamapure S.P.; Letts L.G.; Martino, A.; Ranetunge, R.R.; Schwalb, D.J.; Young, D.V. Lett. Drug Design. Discov. 2005, 2, 40.

19. Ranetunge, R.R.; Augustyniak, M.; Bandarage, U.K.; Earl, R.F.; Murty, M.G.; Richardson, S.R.; Schrodar, J.D.; Shamway, M.J.; Tann, S.W.; Troche A.M.; Young, D.V. J. Med. Chem. 2004, 47, 2180.

20. Vicini, P.; Zani, F.; Cozzini, P.; Doytchinova, I. Eur. J. Med. Chem. 2002, 37, 553.

21. Loncle, C.; Brunel, J.M.; Vidal, N.; Dherbomez, M.; Letourneux, Y. Eur. J. Med. Chem. 2004, 39, 1067.

22. Todeschini, A.R.; Miranda, A.L.P.; Silva, K.C.M.; Parrini, S.C.; Barreiro, E.J. Eur. J. Med. Chem. 1998, 33, 189.

23. Melnyk, P.; Leroux, V.; Sergheraert, C.; Grellier, P. Bioorg. Med. Chem. Lett. 2006, 16, 31.

24. Sridhar, S.K.; Pandeya, S.N.; Stables, J.P.; Atmakuru, R. Eur. J. Pharm. Sci. 2002, 16, 129.

25. Kaymakçloglu, B.K.; Rollas, S. IL Farmaco 2002, 57, 595.

26. Patole, J.; Sandbhor U.; Padhye, S.; Deobagkar, D.N.; Anson, C.E.; Powell, A. Bioorg. Med. Chem. Lett. 2003, 13, 51.

27. Chalina, E.G.; Chakarova, L. Eur. J. Med .Chem. 1998, 33, 975.

28. Delawar, D.L.; Sharma, M.S.; Iyengar, B.S.; Remers, W. A. Antibiot. 1986, 39, 201.

29. Wang, C.L.; Yang, J.F.; Chen, S.Y.; Li, B.Q. Chin. J. Appl. Chem. 2009, 26, 251 and 962.

30. Farghaly, A.R.; El-Kashef, H. ARKIVOK, 2006, 9, 76.

31. Elliot, E.D. J. Amer. Chem. Soc. 1951, 73, 754.

32. Silverstein, R.M.; Webster, F.X. Spectrometric Identification of Organic Compounds, 6th ed., John Wiley and Sons: New Delhi; 2010.

33. Karpenko, A.V.; Kovelenko, S.I.; Shishkin, O.V. Tetrahedron 2009, 65, 5964.

34. Payard, M.; Paris, J.; Couquelet, J. Trav. Soc. Pharm. Montp. 1976, 36, 115; Chem. Abstr. 1977, 85, 152.

35. Abdel-Wahab, B.F.; Abdel-Aziz, H.A.; Ahmed, E.M. Arch. Pharm. Chem. Life Sci. 2008, $341,734$.

36. Kawangh, F. Analytical Microbiology, Academic Press: New York; 1963.

37. British Pharmacopaeia - II, Biological Assay and Tests, The Stationary Office Ltd.: London; 1998; p A-205. 